\title{
A NOTE ON NONSINGULAR FORMS IN A FINITE FIELD
}

\section{CARLITZ}

1. Let $q=p^{n}, p>2, r$ a fixed integer $\geqq 1$. The writer has shown $\left[1\right.$, Theorem 8.3] that one can construct quadratic forms $Q_{1}, \cdots, Q_{r}$

$$
Q_{h}(x)=\sum_{1}^{r} \alpha_{h i j} x_{i} x_{j} \quad\left(\alpha_{h i j} \in G F(q)\right),
$$

such that

$$
\operatorname{det}\left(\xi_{1} Q_{1}+\cdots+\xi_{r} Q_{r}\right) \neq 0
$$

for arbitrary $\left(\xi_{1}, \cdots, \xi_{r}\right) \neq(0, \cdots, 0), \xi_{i} \in G F(q)$. This result suggests the possibility of finding $r$ homogeneous forms $f_{1}, \cdots, f_{r}$ of degree $m$, where $(q, m)=1$ such that $f_{1}, \cdots, f_{r}$ vanish simultaneously only at $(0, \cdots, 0)$, and secondly

$$
f=\xi_{1} f_{1}+\cdots+\xi_{r} f_{r}
$$$$
\left(\xi_{i} \in G F(q)\right)
$$

has no singular point (except at $(0, \cdots, 0)$ ) for arbitrary $\xi_{i}$ not all zero.

To construct such forms let $\beta$ be a number of $G F\left(q^{r}\right)$ such that $\beta, \beta^{q}, \cdots, \beta^{q-1}$ are linearly independent relative to $G F(q)$; Hensel first proved the existence of such $\beta$. Now put

$$
\phi_{i}(x)=\sum_{j=1}^{r} \beta^{a^{i+j}} x_{i}^{m} \quad(i=1, \cdots, r) .
$$

Let $\gamma_{1}, \cdots, \gamma_{r}$ be numbers of $G F\left(q^{r}\right)$ that are linearly independent relative to $G F(q)$ and put

$$
x_{i}=\sum_{k=1}^{r} \gamma_{k}^{q^{j}} y_{k} \quad(j=1, \cdots, r) .
$$

Define $f_{i}(y)$ by means of

$$
f_{i}(y)=\phi_{i}(x) \quad(i=1, \cdots, r) .
$$

Then in the first place

Received by the editors December 24, 1954. 


$$
\begin{aligned}
f_{i}^{q}(y)=\phi_{i}^{q}(x) & =\sum_{j} \beta^{q^{i+j+1}}\left(\sum_{k} \gamma_{k}^{q^{j+1}} y_{k}^{q}\right)^{m} \\
& =\sum_{i} \beta^{q^{i+j}}\left(\sum_{k} \gamma_{k}^{q^{i}} y_{k}^{q}\right)^{m} \\
& =f_{i}\left(y^{q}\right),
\end{aligned}
$$

so that the coefficients of $f_{i}(y)$ are in $G F(q)$.

Now assume that

$$
f_{1}(y)=\cdots=f_{r}(y)=0
$$

for some $\left(y_{1}, \cdots, y_{r}\right)$. Then by (4) and (6), (7) implies

$$
\sum_{j=1}^{r} \beta^{q^{i+j}} x_{j}^{m}=0 \quad(i=1, \cdots, r) .
$$

But since $\beta, \beta^{q}, \cdots, \beta^{q^{r-1}}$ are linearly independent we have that the determinant

$$
\operatorname{det}\left(\beta^{q^{i+j}}\right) \neq 0 \text {. }
$$

Consequently (8) implies $x_{1}=\cdots=x_{r}=0$ and therefore (7) holds only for $y_{1}=\cdots=y_{r}=0$.

Consider next the form $f(y)$ defined by (3). We have

$$
\frac{\partial f(y)}{\partial y_{i}}=\sum_{i, k} \xi_{j} \frac{\partial \phi_{j}(x)}{\partial x_{k}} \frac{\partial x_{k}}{\partial y_{i}}=m \sum_{i, k} \xi_{j} \beta^{q^{j+k}} x_{k}^{m-1} \gamma_{i}^{q^{k}} .
$$

We assume that

$$
\frac{\partial f(y)}{\partial y_{i}}=0 \quad(i=1, \cdots, r)
$$

for some $\left(y_{1}, \cdots, y_{r}\right) \neq(0, \cdots, 0)$. If we put

$$
\eta_{k}=\sum_{j} \xi_{j} \beta^{q i+k}
$$

then (10) and (11) imply

$$
\sum_{k} \eta_{k} x_{k}^{m-1} \gamma_{i}^{q^{k}}=0 \quad(i=1, \cdots, r) .
$$

But the linear independence of $\gamma_{1}, \cdots, \gamma_{r}$ is equivalent to the nonvanishing of the determinant $\operatorname{det}\left(\gamma_{i}^{q^{k}}\right)$; thus (12) implies

$$
\eta_{k} x_{k}^{m-1}=0 \quad(k=1, \cdots, r) .
$$

Since not all $x_{k}$ vanish it follows that 


$$
\sum_{i} \xi_{j} \beta^{a^{i+k}}=0
$$

for at least one value of $k$. But since $\xi_{j} \in G F(q)$, raising (13) to the $q$ th power it follows that (13) holds for all $k=1, \cdots, r$. But in view of (9) this implies $\xi_{1}=\cdots=\xi_{r}=0$.

We have therefore proved the following

TheOREM 1. Let $(q, m)=1, r \geqq 1$. There exist homogeneous polynomials $f_{1}, \cdots, f_{r}$ of degree $m$ with coefficients in $G F(q)$, that vanish simultaneously only at $(0, \cdots, 0)$ and such that

$$
f=\xi_{1} f_{1}+\cdots+\xi_{r} f_{r} \quad\left(\xi_{i} \in G F(q)\right)
$$

has no singular point (except at $(0, \cdots, 0)$ ).

The condition $\xi_{i} \in G F(q)$ is evidently essential.

2. Returning to the case of quadratic forms, the result in (2) cannot be improved. For given $r+1$ quadratic forms, then

$$
\operatorname{det}\left(\xi_{1} Q_{1}+\cdots+\xi_{r+1} Q_{r+1}\right)
$$

is a polynomial of degree $r$ in the $\xi$. Consequently by a well known theorem of Chevalley [2], the determinant (14) vanishes for some $\left(\xi_{1}, \cdots, \xi_{r+1}\right) \neq(0, \cdots, 0)$.

For the case of arbitrary forms of degree $m$ let us take

$$
f(y)=\xi_{1} f_{1}(y)+\cdots+\xi_{8} f_{s}(y) \quad\left(\xi_{i} \in G F(q)\right)
$$

and consider the Hessian

$$
H_{f}=\operatorname{det}\left(\partial^{2} f / \partial y_{i} \partial y_{j}\right) \quad(i, j=1, \cdots, r)
$$

as a polynomial in the $\xi$ 's, $H_{f}$ is of degree $r(m-2)$. Hence if

$$
s>r(m-2)
$$

Chevalley's theorem applies. We may state

THEOREM 2. Let $f_{1}(y), \cdots, f_{8}(y)$ be arbitrary homogeneous polynomials of degree $m$ with coefficients in $G F(q)$ and let (17) hold. Then for arbitrary $y_{i} \in G F(q)$ there exist $\xi_{i} \in G F(q)$ such that the Hessian $H_{f}$ vanishes at $\left(y_{1}, \cdots, y_{r}\right)$.

\section{REFERENCES}

1. L. Carlitz, Invariant theory of systems of equations in a finite field, Journal d'Analyse Mathématique vol. 3 (1954) pp. 382-413.

2. C. Chevalley, Demonstration d'une hypothèse de M. Artin, Abh. Math. Sem. Hansischen Univ. vol. 11 (1936) pp. 73-75.

DUKE UNIVERsity 